\title{
Simulation of Charge-Equilibration and Acceleration of Solar Energetic Ions
}

\author{
A.F. Barghouty and R.A. Mewaldt \\ California Institute of Technology, Pasadena, CA 91125, USA
}

\begin{abstract}
.
Recent measurements of the mean ionic charge states of solar energetic iron and silicon by SAMPEX and ACE during the large solar events of 1992 November 1 and 1997 November 6 show a mean ionic charge that increases with energy. This feature has implications for the use of the observed charge state as a probe of the coronal electron temperature and density, as well as for models of ion acceleration and transport in the coronal plasma. In this paper, we show results of a nonequilibrium model for the mean ionic charge that includes shock-induced acceleration in addition to chargechanging processes. The model is able to reproduce the general features observed without, however, specifying uniquely the acceleration time and the plasma electron density. Based on our simulations for iron and silicon for the 1992 and 1997 events, and assuming a characteristic shock-acceleration time of $\sim 10 \mathrm{sec}$, our model suggests an equilibrationacceleration site at heights $\sim 1$ solar radius above the solar surface, a density $\sim 10^{9} \mathrm{~cm}^{-3}$, and an electron temperature $\sim 1-1.33 \mathrm{MK}$. For ions with kinetic energy $\gtrsim 30 \mathrm{MeV} /$ nucleon we estimate the amount of coronal material the ions traverse to be $\sim 100 \mu \mathrm{g} / \mathrm{cm}^{2}$.
\end{abstract}

\section{INTRODUCTION}

Solar energetic particles (SEPs) observed near $1 \mathrm{AU}$ are known to belong to one of two classes, one associated with impulsive flare events and the other, gradual events, with interplanetary shocks associated with coronal mass ejections (CMEs). Although the distinction between the two classes of events is sometimes blurred, there are, however, population-discriminating features, including the measured charge states of these ions. For example, iron ions with a mean charge of around 20 are found to be associated with impulsive events (1), whereas those with a mean charge of around 14 (similar to the coronal and solar-wind iron seed population) are typically associated with gradual events (2). These charge states can be characterized by temperatures $\sim 10^{7} \mathrm{MK}$ for impulsive events while the gradual ones indicate a temperature $\sim 1-3 \mathrm{MK}$, typical of the ambient corona. Thus, the measured charge state of SEP ions can be used to help discriminate between gradual and impulsive solar events.

The use of equilibrium ionization temperatures to infer the local plasma temperature from the measured mean charge suggests somewhat different temperatures for different ion species $(3,4,5,6)$. An inferred average temperature $\sim 1-3 \mathrm{MK}$ seems, nonetheless, to be consistent with most measured species. Effects like plasma heating, non-Maxwellian velocity distribution for the electrons, and high relative velocities between the energetic ions and ambient plasma electrons were explored and found unable to account for the variation in the inferred temperature $(3,6)$.

Recent measurements of the charge states of solar energetic ions associated with the large solar events of 1992 November 1 and 1997 November 6 by SAMPEX $(5,7)$ and ACE (8) show an energy dependence of the measured mean charge over the energy range $\sim 0.5-50$ $\mathrm{MeV} /$ nucleon. Higher energy ions, especially for iron and silicon, have a higher mean charge. This feature was not reported in earlier observations by ISEE-3 (2) for the 1978-1979 events which covered a much smaller energy interval near $\sim 1 \mathrm{MeV} /$ nucleon. Clearly an energydependent mean charge will affect the interpretation of inferred plasma temperatures.

To appreciate the significance of this energy dependence of the mean charge, one has to realize that the observed charge states of SEPs are expected to be sensitive indicators of the local density and temperature of the charge-equilibration and acceleration site in the coronal plasma. In the case of SEP ions associated with large gradual events, the acceleration is thought to be by large-scale shocks in the outer corona driven by CMEs (e.g., (9)). The charge state is expected to be established via ion-electron and ion-proton collisions in the ambient dense and hot coronal plasma. The rates that establish the equilibrated charge, or the mean charge, depend sen- 
sitively on both the plasma parameters, its density and temperature, as well as on the energy of the ion.

According to Kahler (10), shock acceleration is most effective at heights $\sim 2$ solar radii above the solar surface, above which the electron density begins to drop appreciably. Hence, the observed energy-dependent mean charge is also expected to reflect conditions of the site. Transport effects, from the local equilibration-acceleration site to near $1 \mathrm{AU}$, due to the much lower temperature and density of the solar wind, are not expected to have any appreciable effect on the energy or the charge state of the SEP ions $(11,12)$. Therefore, the observed charge states of SEP ions near 1 AU can, in principle, be used as indicators of the coronal plasma parameters as well as measures of the characteristics of the acceleration mechanism.

Understanding the physical reasons behind the energy dependence of the mean charge is crucial, then, to our understanding of the acceleration processes in SEP events as well as to our ability to infer the plasma parameters characterizing those events. In this paper, we present simulations for the charge distributions of iron and silicon at different energies and for the mean charge as a function of energy, for the 1992 November 1 and 1997 November 6 events. The simulations are based on a nonequilibrium model that couples the charge-changing and acceleration processes in a dynamic fashion. This coupling can produce an energy-dependent mean charge when, e.g., the timescales for acceleration and ionic equilibration are comparable (13).

This model, as well as that of Ostryakov and Stovpyuk (14), is derived assuming that both plasma and shock parameters are stationary in space and time. This assumption is justified when the charge-changing and acceleration processes act over timescales much shorter than those characterizing changes in the plasma and shock parameters. For example, assuming a charge-equilibration and acceleration timescale of $\sim 10 \mathrm{sec}$, an interplanetery shock propagating at $\sim 10^{3} \mathrm{~km} / \mathrm{s}$ will sample a change in the coronal plasma density of only $\sim 25 \%$. An important characteristic of both models, explored in some detail here, is that under this assumption the local density and the characteristic acceleration time cannot be uniquely specified; only their product can be specified. However, by using other means of characterizing the acceleration time, this feature can be quite useful in placing limits on the plasma density for different events.

In addition to these two models (which we term homogeneous models), two recent suggestions addressing the observed energy-dependence of the mean charge have been put forward. Reames et al. (15) argue that under ionic equilibrium conditions the energy dependence of the mean charge arises from electron stripping in "moderately" dense coronal plasma during acceleration. In our model, ionic equilibrium conditions are treated as a lim- iting case. Mason et al. (16), although with a different focus from ours, suggest that the wide range of ionization states is a result of a mixed seed population; one characteristic of a higher-temperature, thus higher charge state, impulsive solar-flare population with the other being of a lower-temperature, gradual-event population.

\section{DESCRIPTION OF THE MODEL}

The model is developed to follow the evolution of the ions' phase-space distribution function in time and momentum subject to the processes of charge-changing ionization and recombination reactions, and shock-induced acceleration. Due to the assumption of homogeneity, the ions are assumed to leak out of the local acceleration region at a rate parameterized by an escape term. This assumption also allows us to ignore propagation effects from the acceleration region to near $1 \mathrm{AU}$. Upon leakage, the ions' steady-state distribution function is frozen-in, both in charge and momentum.

In a spatially homogeneous acceleration region, the shock is idealized as a static and finite plane shock with its normal parallel to the flow of the coronal plasma. In order to avoid introducing another free parameter in the model for the injection energy of the suprathermal ions, we assume that the thermal ions can be sufficiently scattered in momentum space so as to attain (via the secondorder Fermi process) the Alfven mometum, interact with the shock, and be accelerated to tens of $\mathrm{MeV} /$ nucleon via the first-order Fermi process. These ions are also assumed to be energetic enough to be able to leak out of the acceleration region at an escape rate that depends on their energy as well as the strength of the scattering.

Energy losses are assumed to be due only to the passage of the energetic ions through the plasma. In the homogeneous limit, adiabatic energy loss due to the diverging solar wind plasma is ignored.

The ions' balance equation then takes the form:

$$
\begin{aligned}
\frac{\partial f^{q}}{\partial t}= & \frac{1}{p^{2}} \frac{\partial}{\partial p}\left[p^{2} D_{p p} \frac{\partial f^{q}}{\partial p}\right]-\frac{1}{p^{2}} \frac{\partial}{\partial p}\left[p^{2} \frac{d p}{d t} f^{q}\right] \\
& -\frac{f^{q}}{\tau_{\mathrm{esc}}}+Q_{i r}^{q}(p, t)
\end{aligned}
$$

where $f^{q}(p, t)$ is the phase-space density function of ion with charge $q$ as a function of momentum $p$ at time $t$.

The source and sink function $Q_{i r}^{q}(p, t)$, due to ionization and recombination, is given by:

$$
\begin{aligned}
Q_{i r}^{q}(p, t)= & n_{e}\left[R_{i}^{q-1}(p) f^{q-1}+R_{r}^{q+1}(p) f^{q+1}\right. \\
& \left.-\left(R_{i}^{q}(p) f^{q}+R_{r}^{q}(p) f^{q}\right)\right]
\end{aligned}
$$


where $n_{e}$ is the mean electron density and $R_{i}(p)$ and $R_{r}(p)$ are the momentum-dependent ionization and recombination rates. For energetic ions, charge-changing processes are mainly due to ion-electron and ion-proton collisions. Ion-electron ionization cross sections are estimated using the formalism and parameters of $(17,18)$. For ion-proton collisions the cross sections are estimated using the recently developed formalism of (19). The rates are then calculated from the cross sections by multiplying the cross sections by the relative velocities of the energetic ion and ambient thermal electrons and protons, respectively, and by the ambient electron density of the plasma. Calculated this way, the rates depend on the charge and momentum of the ion in addition to the temperature and density of the plasma.

In Eq. (1) the term $f^{q} / \tau_{\text {esc }}$ describes the rate of escape from the shock acceleration region, where

$$
\mathscr{P}_{\mathrm{esc}}=\frac{\tau_{s}}{\tau_{\mathrm{esc}}}=\frac{4 v_{s}}{\eta v}
$$

is the escape probability per shock crossing (e.g., (20)) with $v_{s}$ being the shock propagation speed, $v$ the ion speed, and $\eta$ is the shock compression ratio. In the rest frame of the plasma,

$$
v_{s}=\left(\frac{3 k_{B} T_{e}}{m_{0} c^{2}}\right)^{1 / 2} c
$$

with $T_{e}$ the electron temperature, $k_{B}$ Boltzmann's constant, $m_{0}$ the nucleon's rest mass, and $c$ the speed of light. The cycle time $\tau_{s}$, or time to complete a crossing of the shock front, is given by

$$
\tau_{s}=4\left(1+\eta^{-2}\right) \frac{D_{\|}}{v_{s} v} .
$$

The diffusion coefficient $D_{\|}$along the magnetic field line can be expressed as (21)

$$
D_{\|}(p ; q)=D_{0} P^{2-\alpha} \beta \frac{B_{0}}{B_{s}},
$$

with $D_{0}$ being a constant expressing the strength of the diffusion, $P$ the ion's rigidity, $\alpha$ the spectral index of the magnetic turbulence, and $\beta=v / c$. The ratio $B_{0} / B_{s}$ is the strength of the magnetic field at $1 \mathrm{AU}$ to that at the acceleration site, typically included to ensure that the particle remains tied to the field line (22). In the hard-sphere approximation (23), the diffusion coefficient in momentum space $D_{p p}$ is related to $D_{\|}$as

$$
D_{p p}(p ; q)=\frac{v_{s w}^{2}}{9 D_{\|}} p^{2}
$$

where $v_{s w}$ is the solar wind speed.

The rate of momentum gain per shock crossing is (20)

$$
\left.\frac{d p}{d t}\right|_{+}=\frac{\zeta v_{s w}^{2}}{4 D_{\|}} p
$$

where $\zeta=(\eta-1) / 6 \eta$. Momentum losses due to passage of the energetic ions through the plasma are assumed to be of the standard form:

$$
\left.\frac{d p}{d t}\right|_{-}=- \text {const } \frac{m_{0}}{p} q^{2} n_{e}\left(4 \ln \frac{p}{m_{0} c}-\ln n_{e}+74.1\right) .
$$

For the numerical solution of the system of equations (1), the initial $f^{q}(p, t=0)=f_{0}^{q}(p)$ is assumed Maxwellian in both $p$ and $q$ characterized by the electron temperature $T_{e}$. The boundary conditions in $p$ are taken to be

$$
\begin{gathered}
f^{q}\left(p=p_{0}, t\right)=f_{0}^{q}\left(p=p_{0}\right), \\
\left.\frac{\partial f^{q}(p, t)}{\partial p}\right|_{p=p_{0}}=\left.\frac{\partial f_{0}^{q}(p)}{\partial p}\right|_{p=p_{0}},
\end{gathered}
$$

where $p_{0}$ is the lower limit for $p$ ( $\left.p_{0}<p_{\text {thermal }}\right)$. Other physically reasonable boundary conditions can be used, e.g., requiring $f^{q}(t)$ to remain finite for all $p(14)$. However, we find our simulations to be insensitive to the exact forms of the boundary conditions in $p$. Shock acceleration is assumed to affect only those ions pre-accelerated by diffusing in momentum space and attaining a momentum $p>p_{A}$, where $p_{A}$ is the Alfvén momentum given by $p_{A}=m_{0} B_{s} / \sqrt{\mu n_{e}}, \mu$ being the magnetic permeability constant.

\section{SIMULATIONS}

In Figs. 1 and 2 we show the simulated steady-state solutions of Eq. (1) for the $\mathrm{Fe}$ and $\mathrm{Si}$ charge distributions and the mean charge as a function of energy during the 1992 November event. For these simulations, based on a best fit for the mean charge observations, the electron temperature is taken to be $1.33 \mathrm{MK}$, the density is $3 \times 10^{8} \mathrm{~cm}^{-3}$, and the spectral index $\alpha$ is assumed to be $5 / 3$. Expressed in units of GV for rigidity, the strength of the diffusion coefficient $D_{0}=10^{19} \mathrm{~cm}^{2} / \mathrm{s}$. The strength of the magnetic field at the acceleration site is taken to be 100 Gauss, for which the Alfvén energy is $\approx 0.25$ $\mathrm{MeV} /$ nucleon.

In Figs. 1 and 2 are plotted the normalized charge distributions at energies of $0.32,3.2$, and $32 \mathrm{MeV} /$ nucleon. This choice of energies spans the range available from present measurements. The smooth dotted curves represent simulated charge distributions using only the first and second moments of the phase-space distribution, i.e., assuming a pure Gaussian. The simulated mean charge is 

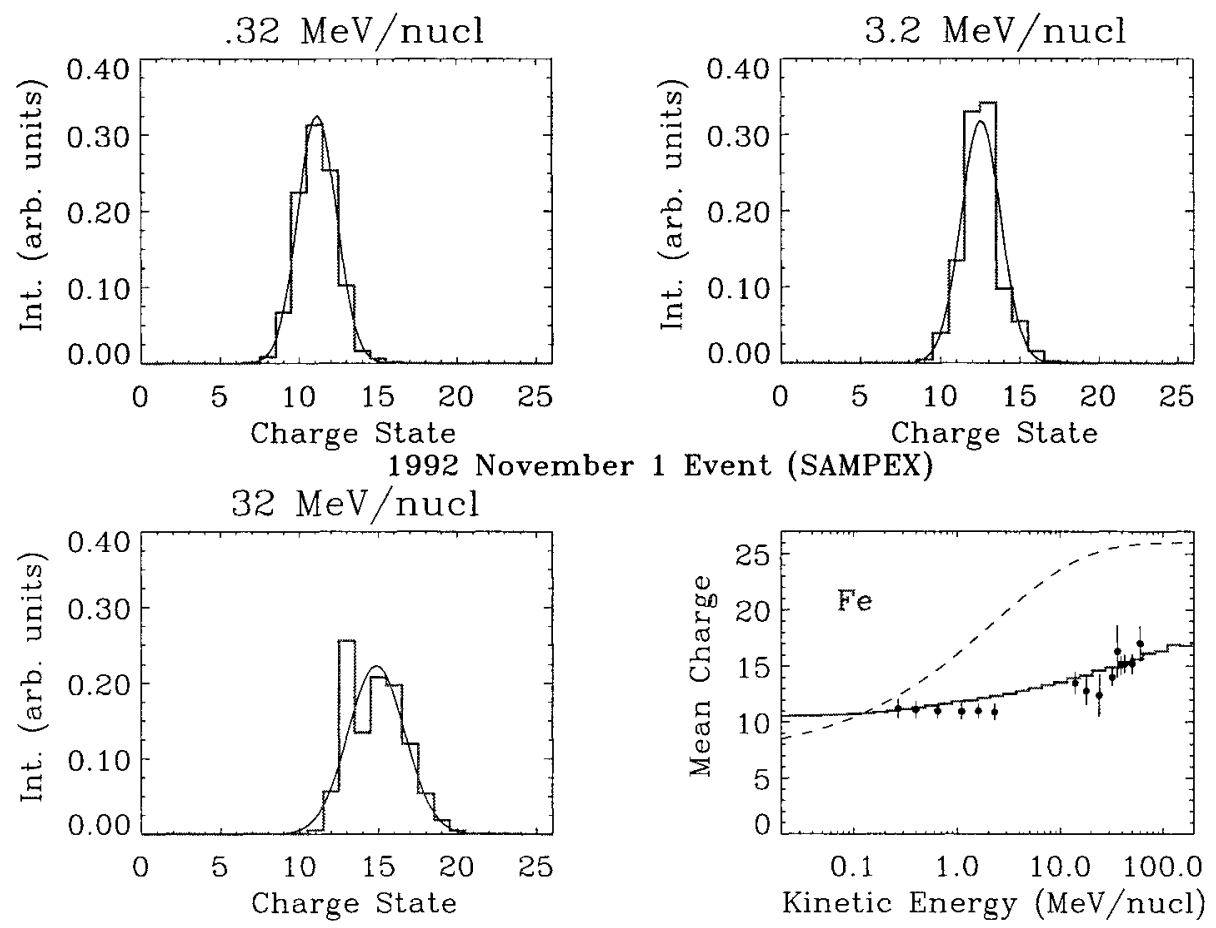

FIGURE 1. Simulated charge distributions (histograms) and mean charge (jagged curve) of iron for the 1992 November event. Solid smooth curves are simulated charge distributions assuming pure Gaussians. The smooth dashed curve is from a semi-empirical formula for the equilibrium mean charge dependence on energy (15). Data points are SAMPEX observations $(4,5,24)$.
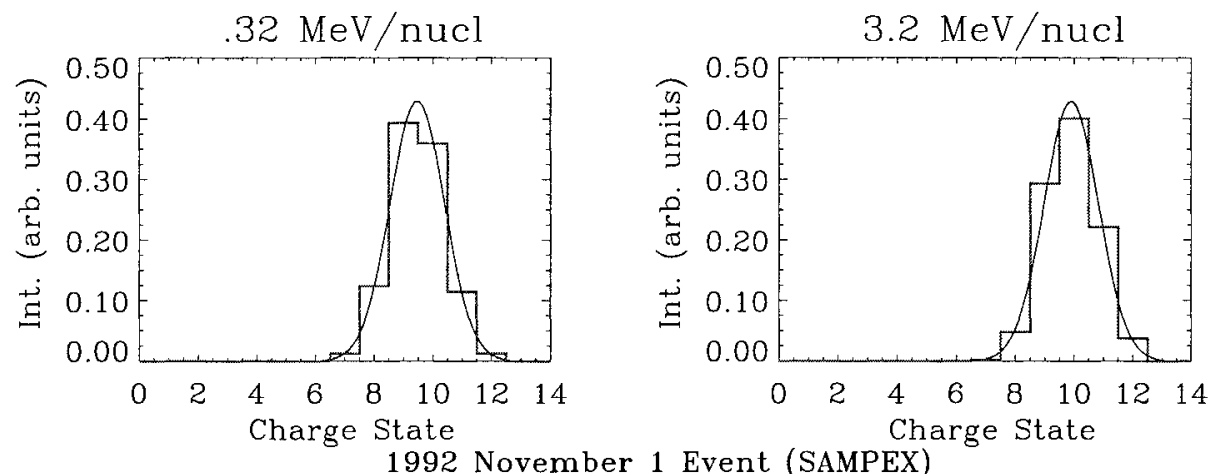

$32 \mathrm{MeV} / \mathrm{nucl}$
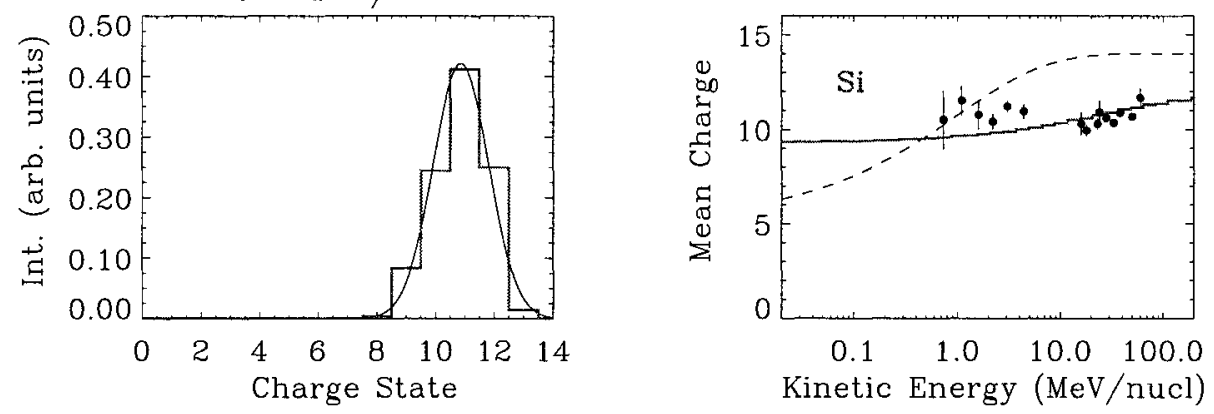

FIGURE 2. Simulated charge distributions and mean charge of silicon for the 1992 November event. [See Fig. 1 and text for further explanation.] 
plotted on the lower right panels (jagged curves). Data points for the mean charge are SAMPEX observations $(4,5,24)$. The dashed smooth curves are the results of a semi-empirical formula for the equilibrium mean charge (15).

The simulations are repeated for the 1997 November event in Figs. 3 and 4. A good fit to the observed mean charge as a function of energy is found for a temperature of $1 \mathrm{MK}$, density $=2 \times 10^{9} \mathrm{~cm}^{-3}$, spectral index $=5 / 3$, and $D_{0}=2 \times 10^{19} \mathrm{~cm}^{2} / \mathrm{s}$. The open-symbol data points for the mean charge are ACE observations (8), while the solid-symbol ones are from SAMPEX (7). (The apparent discrepancy between the ACE and SAMPEX data for the mean charges for the 1997 events is not resolved as of yet.)

Since observations of SEP charge distributions are presently available only for low energy ions $(<1$ $\mathrm{MeV} /$ nucleon)(8), we are deferring detailed discussion of these, as well as energy spectra, to a future paper.

\section{DISCUSSION}

We find that the simulated charge distributions are well approximated by Gaussian distributions with some important qualifications. At low energies, the distribution can depart from a Gaussian due to the lower limit set by the temperature, i.e., the distribution will be skewed toward higher charges. Figs. 3 and 4 at the low energy of $0.32 \mathrm{MeV} /$ nucleon depict this behavior (note that the simulation temperature for this event is lower than that for the 1992 event). The observed charge distributions for iron in the 1997 event by Möbius et al. (8) at comparable energies appear to be similarly skewed. Conversely, at high energies (where no observations of charge state distributions are available yet), the distribution is expected to be skewed toward lower charges due to the upper limit set by the fully stripped ion.

Only for energies well between the two limits do the simulations give distributions closely resembling a pure Gaussian. The third moment (the asymmetry or skewness parameter) of the phase-space distribution is found in our simulations and in (14) to be positive at low energies, cross the zero-line at moderate energies, and be negative at high energies. Deviation from a pure Gaussian at moderate energies can also arise from the structure of the ionization cross sections (e.g., Fig. 3).

The simulated mean charge as a function of energy seen in Figs. 1-4 appears to closely resemble the measured one, but less so for silicon for the 1997 event. It is also clear that both the simulated and measured mean charges in both events do not represent the equilibrium charge state at a given energy (15), even though silicon for the 1997 event appears to be approaching this limit.

For the same Alfvén energy, the simulations shown in Figs. 1-4 are reproduced with different densities and diffusion strengths. We find the results of the simulations unchanged as long as the product $D_{0} \times \sqrt{n_{e}}$ is kept constant. For example, for the 1992 event increasing the density by a factor of 10 to $3 \times 10^{9} \mathrm{~cm}^{-3}$ while decreasing $D_{0}$ to $10^{19} / \sqrt{10} \mathrm{~cm}^{2} / \mathrm{s}$ produces identical results to those shown on Figs. 1 and 2. Or, for the 1997 event decreasing the density by a factor of 10 to $2 \times 10^{8} \mathrm{~cm}^{-3}$ while increasing $D_{0}$ to $2 \sqrt{10} \times 10^{19} \mathrm{~cm}^{2} / \mathrm{s}$ gives identical results to those shown on Figs. 3 and 4.

In this model, the overriding parameters for the simulated mean charge are the magnitude of the diffusion coefficient and the plasma density. Both directly affect the acceleration and equilibration timescales. For both simulated events, even though the two parameters are allowed to vary, the timescale for acceleration remains comparable to the equilibration timescale in those simulations that fit the observed energy dependence of the mean charge. (Other parameters in this model also affect the timescales but less sensitively, e.g., the Alfvén momentum.) Altering the acceleration timescale relative to equilibration appreciably, on the other hand, produces simulated mean charges that do not resemble the measured ones.

Comparable timescales in this homogeneous model translate into charge-equilibration and acceleration processes taking place concurrently. This is equivalent to stating that at a given energy comparable timescales suggest comparable plasma grammage inferred from either process. Since this grammage depends on the ion energy, ions with higher energy traverse more grammage, and vice versa, giving rise to an energy-dependent mean charge. However, the grammage also depends on the product of the plasma density $\times$ the characteristic residence time (for equilibration and acceleration). Below, we explore this further.

The acceleration time $\tau_{\mathrm{acc}}$ is proportional to $D_{0}$ via

$$
\tau_{\mathrm{acc}} \sim \int_{p_{i}}^{p_{c}}\left(\frac{d p}{d t}\right)^{-1} d p,
$$

where $p_{i} \sim p_{A}$ and $p_{c}>p_{i}$, and with $d p / d t \propto 1 / D_{0}$ from Eqs. (6) and (8). The residence or escape time $\tau_{\text {esc }}$ and equilibration time (time to reach ionic equilibrium) $\tau_{\mathrm{eq}}$ are related to the amount of coronal material the ions encounter as

$$
\lambda_{\text {esc }} \propto \tau_{\text {esc }} v n_{e}, \lambda_{\text {eql }} \propto \tau_{\text {eq1 }} v n_{e} .
$$

Now, from Eq. (1) in steady-state, and for a fixed Alfvén momentum, a sufficient condition for the mean charge to be an increasing (but bounded) function of momentum (i.e., $d\langle q\rangle / d p>0$ ) is 

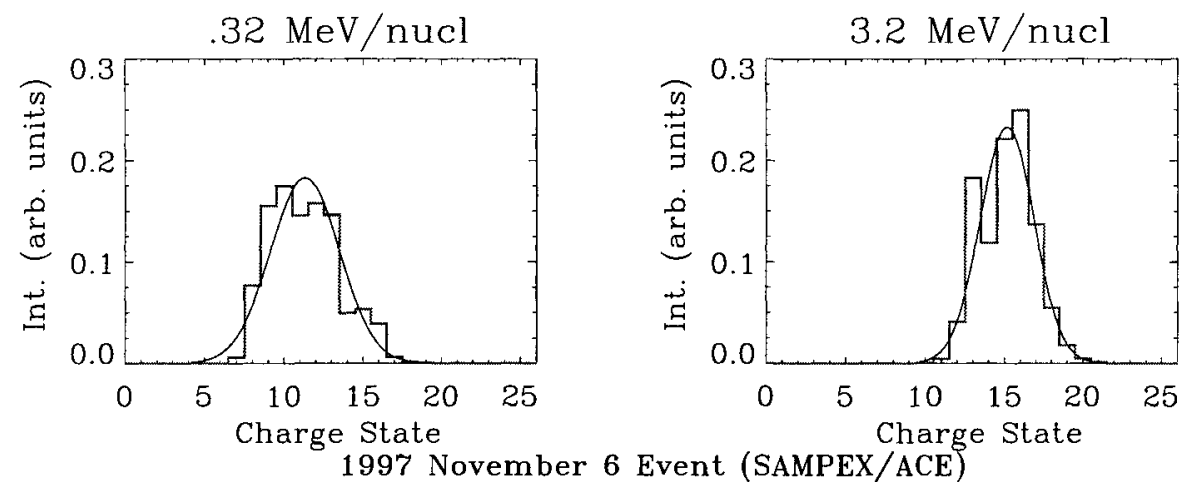

$32 \mathrm{MeV} / \mathrm{nucl}$
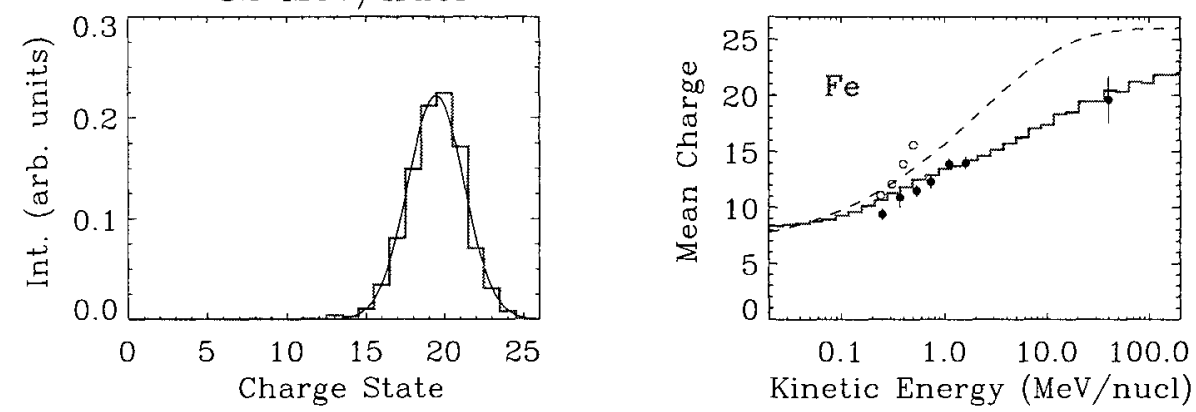

FIGURE 3. Simulated charge distributions and mean charge of iron for the 1997 November event. Solid data points are SAMPEX observations (7) while open ones are ACE observations (8). [See also Fig. 1.]
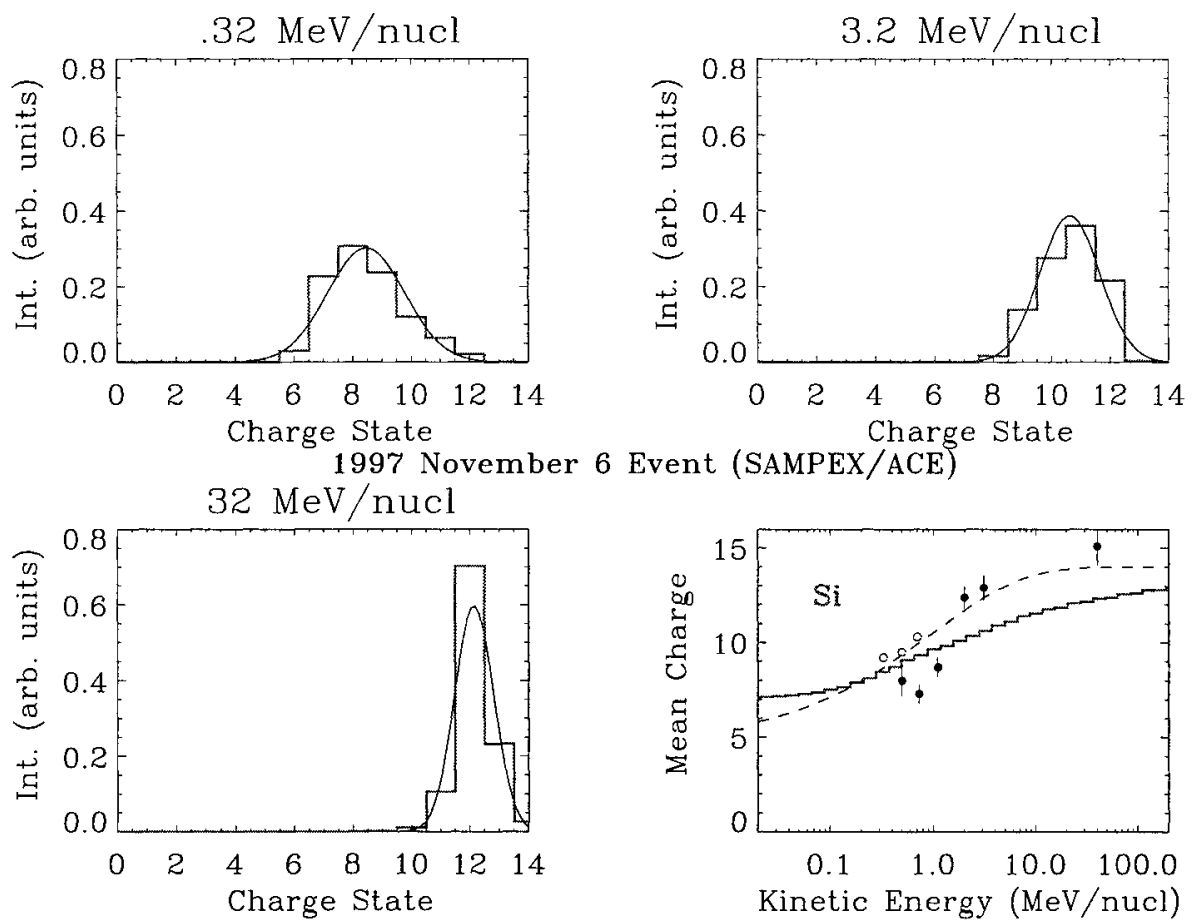

FIGURE 4. Simulated charge distributions and mean charge of silicon for the 1992 November event. [See Fig. 3 and text for further explanation.] 


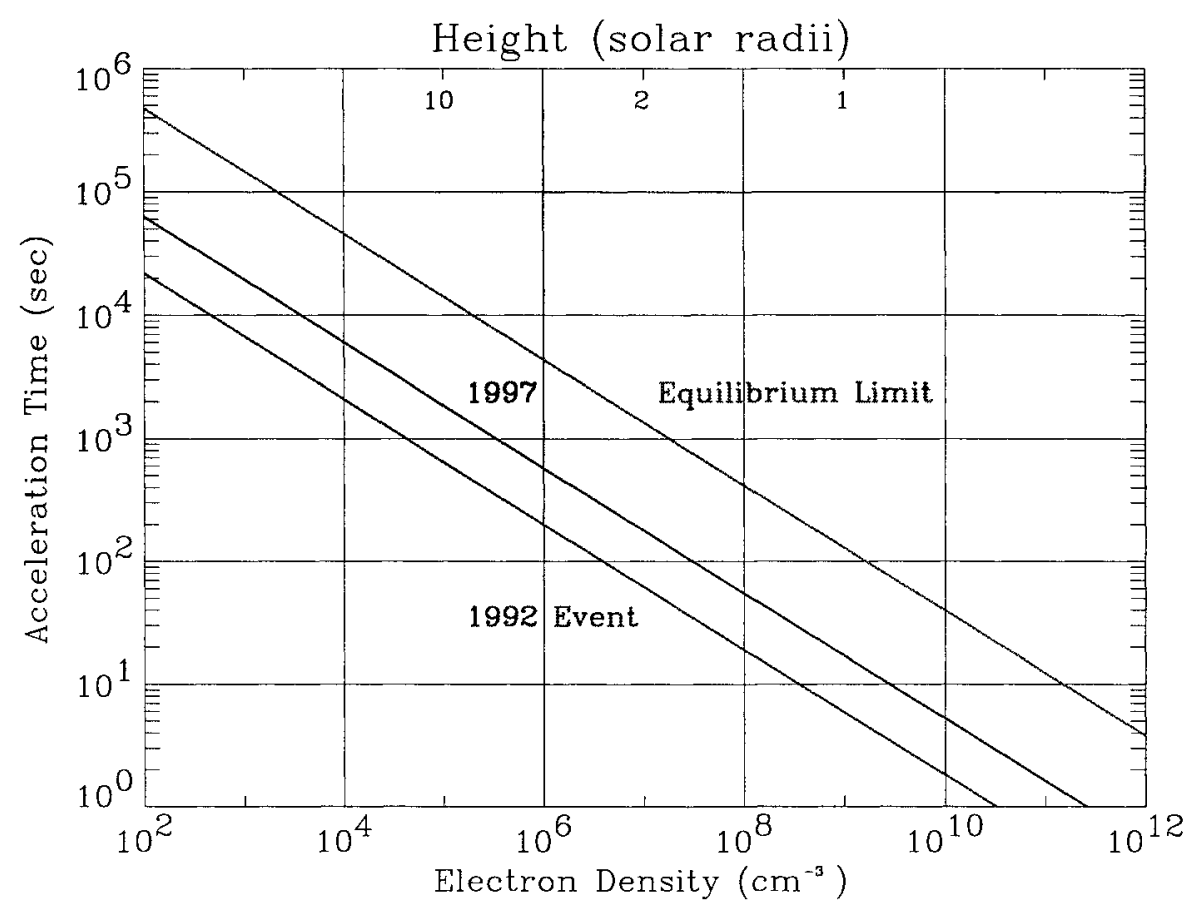

FIGURE 5. Calculated loci of acceleration time vs. electron density (or height above the solar surface) for the 1992- and 1997-events simulations. These loci are for ions with kinetic energy $\gtrsim 30 \mathrm{MeV} /$ nucleon characterized by a constant acceleration time $\times \sqrt{ }$ plasma electron density. The "equilibrium limit" depicts charge-state equilibrium (15). All points on the 1992-event locus, for example, give the same results shown on Figs. 1 and 2.

$$
\lambda_{\text {esc }}^{-1}-\lambda_{\text {eql }}^{-1}>\frac{\text { const }}{\tau_{\mathrm{acc}} \sqrt{n_{e}}}
$$

Fig. 5 depicts the loci of the acceleration time vs. electron density for iron and silicon ions with kinetic energy $\gtrsim 30 \mathrm{MeV} /$ nucleon for the 1992 and 1997 events, as well as for equilibrium conditions (15) in which, at these energies, both ions are fully stripped of their electrons. All three loci on Fig. 5 are characterized by a constant $\tau_{\text {acc }} \times \sqrt{n_{e}}$, e.g., all points on the 1992-event locus give the same results shown on Figs. 1 and 2. In Fig. 5 height above the solar surface is calculated assuming hydrostatic equilibrium with $T_{e}=1.2 \mathrm{MK}$ and with density varying with height $h$ as (25)

$$
n_{e}=4.2 \times 10^{4+4.32 / h} .
$$

For the 1997 event an upper limit for the acceleration time can be estimated from the time interval from when SOHO first observed the CME (12:10 UT) to when ACE first observed $\gtrsim 30 \mathrm{MeV} /$ nucleon ions at L1 (13:00 UT), corrected for the transit time. This gives an estimate for the acceleration time of $\sim 10 \mathrm{~min}$. From Fig. 5, the corrsponding lower limit for density for the 1997 event is then $\sim 10^{6} \mathrm{~cm}^{-3}$, and the corresponding upper limit for height $\sim 3$ solar radii.
Although we cannot specify a more precise acceleration time from these results alone, we can use measurements of hard X-rays and $\gamma$-rays from other SEP events to infer a timescale. Time-profile measurements of hard X-ray and $\gamma$-ray emission are known to depend sensitively on electron and ion acceleration time (e.g., $(26,27))$. For protons and ions accelerated to energies $\gtrsim 10 \mathrm{MeV} /$ nucleon, a characteristic acceleration time of $\lesssim 10 \mathrm{sec}$ can be inferred from the measured time-profile of the X-ray intensity $(27,28)$. Although this timescale is expected to vary from one event to another, below we assume an acceleration timescale of $\sim 10 \mathrm{sec}$ to characterize the 1992 and 1997 events.

With a characteristic acceleration time $\sim 10 \mathrm{sec}$ for ions with energy $z 30 \mathrm{MeV} /$ nucleon, our model (see Fig. 5 ) is consistent with an equilibration-acceleration site at heights $\sim 1$ solar radii above the solar surface, where the density is $\sim 10^{9} \mathrm{~cm}^{-3}$ and the typical temperature is $\sim 1.0-1.33 \mathrm{MK}$. Based on our simulations for the 1992 and 1997 events, we estimate that for these ions the amount of coronal material traversed during equilibration and acceleration is $\sim 100 \mu \mathrm{g} / \mathrm{cm}^{2}$. On the other hand, equilibrium conditions (15) suggest a grammage of $\sim 10$ $\mathrm{mg} / \mathrm{cm}^{2}$ and densities of $\sim 10^{11} \mathrm{~cm}^{-3}$, which is typical of the lower corona at heights of $\sim 0.1$ solar radius. 


\section{SUMMARY}

We have presented simulations for the mean charge and charge distributions of iron and silicon ions for the 1992 November 1 and 1997 November 6 large particle events. Measurements of the mean charge state of solar energetic ions in these events have shown that the mean charge increases with energy; a feature that has not been seen before. Understanding the physical reasons for this new feature is critical to our ability to infer the local coronal plasma temperature and density, as well as to characterize and model the acceleration process.

The simulations presented here are based on a nonequilibrium model that couples the processes of charge-changing and acceleration in a dynamic fashion. In this model plasma and shock parameters are assumed stationary in space and time (the homogeneous limit). The model is able to reproduce the general features of the mean charge as a function of energy seen in the 1992 and 1997 events. We find that ionic equilibrium conditions to be a limiting case in this model, and that neither event appears to correspond to ionic equilibrium.

The simulations suggest that the energy dependence of the mean charge is due to the charge-changing and acceleration processes taking place concurrently. In the homogeneous limit in which this model is derived, this is equivalent to stating that at a given energy the amount of coronal material that the ions encounter inferred from either process is the same. Since this grammage depends on the ion energy, ions with higher energy traverse more grammage, and vice versa, giving rise to an energy-dependent mean charge.

The simulations have also shown that a characteristic of models derived in this limit is the ability to specify the plasma grammage without being able to specify uniquely the acceleration time or the electron density of the plasma; lacking additional information only the product $\tau_{\text {acc }} \times \sqrt{n_{e}}$ can be specified. However, we do find the range of this product consistent with a wide range of solar-wind conditions.

In order to infer the location of the equilibrationacceleration region, we need to specify the acceleration timescale. This timescale can be inferred from hard Xrays and $\gamma$-rays from SEP events. Assuming a characteristic shock-acceleration time of $\sim 10 \mathrm{sec}$, and based on our simulations for the 1992 and 1997 events, this model is consistent with an equilibration-acceleration site at heights $\sim 1$ solar radius above the solar surface, electron temperatures $\sim 1-1.33 \mathrm{MK}$, and plasma electron densities $\sim 10^{9} \mathrm{~cm}^{-3}$. Our calculations also suggest that for ions with kinetic energy $\gtrsim 30 \mathrm{MeV} /$ nucleon the amount of coronal material the ions traverse during equilibration and acceleration is $\sim 100 \mu \mathrm{g} / \mathrm{cm}^{2}$.

\section{ACKNOWLEDGMENTS}

Work is supported by NSF grant 9810653 and NASAJOVE NAG8-1208 and by NASA grants NAS5-30704 and NAG5-6912 at Caltech. A.F.B. thanks Prof. M. Yoshimori for stimulating discussions and Dr. Christina Cohen for valuable help with SOHO and ACE data.

\section{REFERENCES}

1. A. Luhn et al., Astrophys. J. 317, 951 (1987).

2. A. Luhn et al., Adv. Space Res. 4, 161 (1984).

3. A. Luhn and D. Hovestadt, Proc. 19th Int. Cosmic-Ray Conf. (La Jolla) 4, 245 (1985).

4. R.A. Leske et al., Astrophys. J. Lett. 452, L149 (1995).

5. M. Oetliker et al., Astrophys. J. 477, 495 (1997).

6. D. Ruffolo, Astrophys. J. Lett. 481, L119 (1997).

7. J.E. Mazur et al., Geophys. Res. Lett. 26, 173 (1999).

8. E.M. Möbius et al., Geophys. Res. Lett. 26, 145 (1999).

9. D.V. Reames, Astrophys. J. Lett. 358, L63 (1990).

10. S.W. Kahler, Astrophys. J. 428, 837 (1994).

11. D. Hovestadt et al., Astrophys. J. 281, 463 (1984).

12. A. Luhn and D. Hovestadt, Astrophys. J. 317, 852 (1987).

13. A.F. Barghouty and R.A. Mewaldt, Astrophys. J. Lett. 520, L127 (1999).

14. V.M. Ostryakov and M.F. Stovpyuk, Solar Phys. 189, 357 (1999).

15. D.V. Reames, C.K. Ng, and A.J. Tylka, Geophys. Res. Lett. 26, 3585 (1999).

16. G.M. Mason, J.E. Mazur, and J.R. Dwyer, Astrophys. J. Lett. 525, L133 (1999).

17. M. Arnaud and J. Raymond, Astrophys. J. 398, 394 (1992).

18. M. Arnaud and R. Rothenflug, Astron. and Astrophys. 60, 425 (1985).

19. A.F. Barghouty, Phys. Rev. A 61, 052702 (2000).

20. M.A. Forman and G.M. Webb, in Collisionless Shocks in the Heliosphere: A Tutorial Review, eds. R.G. Stone and B. Tsurutani (Geophys. Monog. 34), (AGU: Washington, DC, 1985), 91.

21. J.R. Jokipii, Rev. Geophys. Space Phys. 9, 27 (1971).

22. J.R. Jokipii and G. Morfill, Astrophys. J. 312, 170 (1987).

23. J. Skilling, Mon. Not. R. Astron. Soc. 172, 557 (1975).

24. G.M. Mason et al., Astrophys. J. 452, 901 (1995).

25. H. Zirin, Astrophysics of the Sun, (Cambridge U. Press: Cambridge, England, 1989), 226.

26. S.R. Kane et al., Astrophys. J. Lett. 300, L95 (1986).

27. E.L. Chupp, Ann. Rev. Astron. Astrophys. 22, 359 (1994).

28. M. Yoshimori et al., Adv. Space Res. 25, 1801 (2000). 